\title{
Balkanologie
}

Balkanologie Revue d'études pluridisciplinaires

Vol. VIII, $n^{\circ} 1$ | 2004

Volume VIII Numéro 1

\section{Autochtones étrangers : les déplacés à Mostar après la guerre de Bosnie-Herzégovine}

Foreign natives: the displaced people in Mostar after the war in Bosnia-

Herzegovina

\section{Stéphanie Rolland}

\section{OpenEdition}

\section{Journals}

Édition électronique

URL : http://journals.openedition.org/balkanologie/526

DOI : $10.4000 /$ balkanologie. 526

ISSN : 1965-0582

Éditeur

Association française d'études sur les Balkans (Afebalk)

Édition imprimée

Date de publication : 1 juin 2004

ISSN : 1279-7952

\section{Référence électronique}

Stéphanie Rolland, «Autochtones étrangers : les déplacés à Mostar après la guerre de Bosnie-

Herzégovine », Balkanologie [En ligne], Vol. VIII, n 1 | 2004, mis en ligne le 21 janvier 2010, consulté le

17 décembre 2020. URL : http://journals.openedition.org/balkanologie/526 ; DOI : https://doi.org/

10.4000/balkanologie.526

(c) Tous droits réservés 


\title{
AUTOCHTONES ÉTRANGERS : LES DÉPLACÉS À MOSTAR APRÈS LA GUERRE DE BOSNIE-HERZÉGOVINE
}

\author{
Stéphanie Rolland*
}

La pratique du nettoyage ethnique pendant le conflit bosniaque de 19921995 a entraîné de vastes mouvements de population. Ainsi, d'après les estimations du Haut Commissariat aux Réfugiés des Nations unies, 1100000 personnes se sont réfugiées ${ }^{1}$ principalement dans les anciennes républiques yougoslaves voisines, au sein de la Communauté européenne et dans les pays du Proche-Orient, tandis qu'un million à un 1300000 personnes ont été déplacées à l'intérieur même des frontières de la Bosnie-Herzégovine, sur une population totale de quatre millions et demi d'habitants. La signature des accords de paix de Dayton en Novembre 1995 a mis fin à la guerre entre Bochniaques, Croates et Serbes ${ }^{2}$, en divisant la République de Bosnie-Herzégovine en deux

'Doctorante en Anthropologie à l'Université de Provence et rattachée au laboratoire de recherche IDEMEC de la Maison Méditerranéenne des Sciences de l'Homme d’Aix-en-Provence. (steph_rk@hotmail.com).

${ }^{1}$ Le UNHCR établit la distinction entre la catégorie "réfugié n (" refugee "), personne qui a trouvé asile à l'étranger, et " déplacé " ( DP, Displaced Person ", ou " IDP, Internally Displaced Person n) qui a trouvé refuge à l'intérieur des frontières de son pays. De même, il existe la distinction entre " réfugié de retour " ( refugee returnee ") dans son pays d'origine, et " déplacé de retour " ("DP returnee n) dans son habitation d'origine. Il arrive que des réfugiés de retour se trouvent désormais en situation de déplacés.

${ }^{2}$ Le terme " bosniaque " ( bosanac/pluriel : bosanci ", adjectif " bosanski $n$ ) désigne l'ensemble des habitants de la Bosnie-Herzégovine, quelle que soit leur appartenance ethno-nationale. La Constitution de la nouvelle République de Bosnie-Herzégovine reconnaît trois peuples constitutifs égaux en droits : les Bochniaques, "Bošnjaci ", les Croates, "Hrvati ", et les Serbes, "Srbi ", ainsi que trois langues : bosniaque (" bosanski jezik "), croate ( hrvatski jezik ") et serbe ("srpski jezik »). Les Bochniaques, désignés par le terme "Musulmans " ("Muslimani ") à l'époque de la Yougoslavie, sont les Bosniaques de tradition religieuse musulmane (les Croates sont de tradition religieuse catholique et les Serbes de tradition religieuse orthodoxe). Nous avons choisi d'emprunter la traduction phonétique " bochniaque n de D. Dimitrijević (Dimitrijevic (Dejan), " Ajvatovica. Analyse de la tentative de construction d'un mythe fondateur de l'identité bochniaque ", Annales de la Fondation Fyssen, (13), 1999 ) pour l'ethnonyme "Bošnjak» afin de respecter la nuance qui existe dans la langue vernaculaire entre "bosanac/bosanci/bosanski " et "bošnjak/bošnjac/bošnjaćki n. 
entités politiques autonomes : la République serbe, qui englobe $49 \%$ du territoire, et la Fédération de Bosnie-Herzégovine ou Fédération bosno-croate, 51 $\%$ du territoire. Cette partition officielle a provoqué de nouveaux mouvements de population, notamment des déplacements spontanés de Serbes de la Fédération vers la République serbe ${ }^{3}$. Fin 2002, 7 ans après l'arrêt du conflit, le nombre de déplacés s'élevait encore à 403000 personnes. C'est en République serbe que les retours sont les plus difficiles et les moins nombreux, ainsi que dans certaines villes de la Fédération comme Mostar. Le nombre encore élevé de déplacés en Bosnie-Herzégovine et leur situation illustrent les conséquences quotidiennes de la guerre et de la pratique du nettoyage ethnique sur les habitants du pays, c'est-à-dire la perpétuation sournoise de la violence engendrée par le conflit et les difficultés de retour à une vie normale dans un pays en reconstruction où la crise économique semble interminable.

\section{MOSTAR : UN DÉPLACÉ POUR DEUX HABITANTS}

Deux facteurs avaient contribué à faire de Mostar le symbole de la BosnieHerzégovine multiethnique : d'une part l'hétérogénéité de sa population (un tiers de Bochniaques, un tiers de Croates et un cinquième de Serbes sur 126600 habitants 4 ) et d'autre part le nombre élevé de mariages mixtes (entre Bosniaques et Herzégoviniens de différente nationalité) 5 . Après la guerre de 1992-1995, la ville est devenue l'archétype de la Bosnie-Herzégovine divisée et du repli communautaire des principales composantes ethno-nationales du pays. Mostar a subi deux conflits distincts pendant les années de guerre. Le premier correspond à l'attaque de l'armée nationale yougoslave (Jugoslovenska Narodna Armija - JNA) sous contrôle serbo-monténégrin en avril 1992 qui a entraîné la fuite massive des Serbes de Mostar principalement en Herzégovine orientale, et l'exil temporaire de femmes et d'enfants bochniaques et croates en Croatie, sur la côte dalmate toute proche, jusqu'au retrait

\footnotetext{
${ }^{3}$ Les estimations de l'UNHCR pour l'année 1996 font état de 80 ooo nouveaux déplacés dont 60 ooo Serbes de la Fédération vers la République serbe au moment des transferts de territoires entre les deux entités, consécutifs à l'application des accords de Dayton (novembre-décembre 1995). Entre la signature des accords de paix fin 1995 et 1999, le nombre de personnes déplacées après la guerre s'élève à 200000 , parmi lesquels de nombreux réfugiés de retour qui n'ont pas pu réintégrer leur habitation d'avantguerre.

4 D'après le dernier recensement yougoslave effectué en 1991.

5 À l'époque de la République Socialiste Fédérale de Yougoslavie, la Bosnie-Herzégovine était la seule république où aucun groupe national ne représentait la majorité absolue de la population : le recensement de 1991 faisait état de $43 \%$ de Musulmans (désormais appelés Bochniaques), $31 \%$ de Serbes, $18 \%$ de Croates et $8 \%$ d'autres minorités ethniques et nationales, d'après les catégories nationales définies par le régime yougoslave.
} 
de la INA fin juillet. À la même époque, les leaders nationalistes croates de Bosnie-Herzégovine constituent une communauté autonome croate du nom d'Herzeg-Bosnie, proclamée république avec Mostar pour capitale fin août 1993. Le deuxième conflit éclate en mai 1993, cette fois-ci entre Bochniaques et Croates. La ville se scinde alors définitivement en deux : la partie est, à majorité bochniaque est enclavée et les Bochniaques résidant à l'ouest sont massivement expulsés de leur logement, de même que les Croates résidant à l'est. Un nombre important de Mostariens se trouve ainsi en position de déplacés dans leur propre ville, contraints à s'installer dans les habitations délaissées par leurs co-citadins. Certains se réfugient à l'étranger momentanément, d'autres définitivement, en particulier les couples mixtes pour lesquels il était impossible de " choisir un camp " plutôt qu'un autre. Comme jamais dans son histoire, la ville est divisée ethniquement entre Bochniaques à l'est et Croates à l'ouest. En même temps que la moitié des habitants d'avant-guerre abandonne la ville, Mostar voit déferler des vagues successives de déplacés bochniaques et croates, d'origine rurale ou de petites villes provinciales, qui tentent d'échapper au nettoyage ethnique.

La fondation de la ville par les Ottomans, son développement au cours des siècles et l'explosion urbaine au lendemain de la seconde guerre mondiale sous l'impulsion de l'essor industriel ont contribué à ce que la partie est (la plus ancienne), ait depuis toujours été habitée par une majorité de Bochniaques, alors que la population résidant dans la partie ouest récente était beaucoup plus hétérogène. La configuration géographique de la vallée de la Neretva autour de Mostar a conditionné l'extension de la nouvelle ville sur la rive droite, alors que la vieille ville s'étend sur la rive gauche et sur une fine bande de la rive droite, entre la rivière et le boulevard. C'est pourquoi l'essentiel des infrastructures, hôpital, stade, université, etc., se situait à l'ouest. Par conséquent, la partition de la ville en deux a obligé les Bochniaques à improviser d'urgence, en plein conflit, les infrastructures qui faisaient défaut. La division entre Bochniaques et Croates a imposé une duplication institutionnelle encore dominante à tous les niveaux. Depuis l'arrêt du conflit, la frontière désormais virtuelle entre l'est et l'ouest de la ville est demeurée l'ancienne ligne de front, matérialisée par le bulevar qui traverse la ville dans un axe nord-sud parallèle à la rivière Neretva.

Aujourd'hui, malgré la fin de la guerre et les efforts de la communauté internationale pour réunifier la ville et ses habitants, Mostar fonctionne toujours comme si deux États cohabitaient dans la même ville, selon un principe d'organisation communautariste à base ethno-nationale : la ville est administrativement divisée en six municipalités, trois à majorité bochniaque et trois à majorité croate, universités et écoles sont divisées, de même que les hôpitaux, les services des postes et des télécommunications, les services des eaux, les caisses de retraite, les tribunaux, les médias, etc. Bien que la communauté internationale ait imposé un commandement commun aux polices et aux 
armées, casernes et commissariats sont encore séparés. Mais, la division de la ville entre Bochniaques et Croates masque une autre division fondamentale : la division entre anciens habitants et déplacés. Depuis quelques années, des déplacés originaires de Mostar et des villages environnants sont rentrés chez eux, mais ils sont devenus des étrangers dans leur propre ville ou village. Les relations entre habitants d'origine et déplacés ainsi qu'entre nouveaux habitants (déplacés) et déplacés de retour apportent une nuance significative au syndrome de la ségrégation ethno-nationale du pays.

\section{LES DÉPLACÉS : L'INTÉGRATION MANQUUÉE}

À Mostar, la signature des accords de paix n'a pas pour autant mis un terme à l'expulsion des Bochniaques qui résidaient encore à l'ouest, et quelques 100 personnes ont été expulsées au courant de l'année 1996. Depuis $2001-2002^{6}$, les Bochniaques de Mostar ont été nombreux à récupérer leur appartement à l'ouest, mais ils cherchent parfois à l'échanger contre un appartement " croate " situé à l'est ou décident de vendre lorsqu'ils ont définitivement émigré à l'étranger. D'autres encore, après avoir été réfugiés à l'étranger, sont désormais déplacés dans leur propre ville, en attente de la restitution d'un appartement occupé par d'autres déplacés.

D'après un recensement de l'UNHCR réalisé en 1998, trois ans après la fin de la guerre, la population de Mostar était composée de $53 \%$ de Bochniaques dont plus de la moitié $(55,4 \%$ ) étaient des déplacés, de $45 \%$ de Croates, dont plus d'un tiers de déplacés (34,7\%), et de $2 \%$ de Serbes ; $89 \%$ des Bochniaques résidaient à l'est et $11 \%$ à l'ouest, $96 \%$ des Croates résidaient à l'ouest et $4 \%$ à l'est, $80 \%$ des Serbes résidaient à l'ouest et $20 \%$ à l'est. Les Bochniaques restés à l'ouest pendant la guerre sont originaires de Mostar et rarement en couples mixtes, tandis que les Croates et les Serbes résidant à l'est sont presque tous des époux de couples mixtes bochniaque-croate ou bochniaque-serbe. L'arrivée massive des déplacés à Mostar pendant la guerre a non seulement profondément modifié la structure sociale et ethno-nationale de la ville mais aussi renforcé les clivages qui existaient déjà dans la société yougoslave d'autrefois entre les mondes rural et urbain.

Les déplacés bochniaques de Mostar viennent essentiellement de petits centres urbains et de villages d'Herzégovine orientale désormais englobés dans la République serbe (triangle Nevesinje, Gacko, Trebinje) et du sud de l'Herzégovine sous contrôle croate (région de Stolac et Čapljina). Les déplacés croates viennent quant à eux principalement du nord de l'Herzégovine (région

${ }^{6}$ Pour des raisons de cohérence, le terrain correspondant ayant pris fin en décembre 2001, l'utilisation des données établies par les organismes internationaux se limite à 2002. 
de Konjic et vallée de la Neretva sous contrôle bochniaque), de Bosnie centrale (région de Kiseljak) et quelques uns de Sarajevo : " Ici on appelle Mostar ouest Ki-Ko car les réfugiés croates viennent tous des environs de Kiseljak et de Konjic $"$ ?.

Les traitements de faveur à l'égard des déplacés, croates en particulier en ce qui concerne l'attribution des parcelles de terrain constructible et de l'aide à la reconstruction, suscite un fort sentiment d'injustice chez les Mostariens :

Là-bas, en bas [elle désigne le nouveau lotissement construit au sud de Mostar, juste après l'aéroport], ce sont toutes des maisons de réfugiés croates, le terrain leur a été donné par la municipalité et le matériel a été financé par le gouvernement de Tudjman ; nous, la maison était à peine terminée quand la guerre a commencé, elle a été endommagée, mais nous n'avons rien reçu, aucune aide, nous avons tout reconstruit avec notre argent ${ }^{8}$.

En effet, les Croates de Bosnie-Herzégovine ont bénéficié d'un important soutien financier de la Croatie jusqu'à la mort du président Tudjman en décembre 1999. Le gouvernement croate a financé la construction de hameaux entiers dans les zones sous contrôle croate, en Herzégovine du sud dans le triangle Mostar (municipalité sud à majorité croate) - Stolac - Čapljina, favorisant l'installation définitive des déplacés afin de maintenir cette région ethniquement homogène. Ils ont été baptisés par leurs nouveaux habitants " village de Tudjman " (Tuđtmanovo selo), " village de Boban9 "(Bobanovo selo) ou encore " village de Šušak ${ }^{10}$ " (Šuškovo selo). Le projet du ministre de la Défense croate de l'époque était de créer une large bande territoriale à majorité croate en Herzégovine occidentale, parsemée de nouveaux villages afin de renforcer la frontière de la Croatie ${ }^{11}$. C'est pour cette raison qu'ils se situent principalement sur la rive droite de la Neretva, le long de l'axe routier qui relie Mostar à la côte dalmate, à proximité des points stratégiques comme l'aéroport de Mostar et dans les zones de retour potentiel de déplacés bochniaques et serbes. Deux lotissements de ce type sont distants de quelques kilomètres seulement, sur le côté ouest de la nationale qui mène en Croatie, près de l'aéroport à Ortiješ (en face du camp militaire de la $\mathrm{SFOR}^{12}$ ) et à Buna. Les parcelles et le

\footnotetext{
${ }^{7}$ Mirjana, croate épouse d'un Bochniaque, résidante ả l'est, 70 ans.

${ }^{8}$ Vera, croate, 50 ans.

9 du nom d'un général de l'armée croate HVO-BiH et président de l'Herzeg-Bosnie, Mate Boban.

${ }^{10}$ D'après Gojko Šušak, Ministre de la Défense Croate pendant la guerre et planificateur de l'implantation des déplacés croates dans les territoires à majorité croate.
}

${ }^{11}$ D'après une circulaire interne de l'OHR (Office of High Representative), " From Dayton to Europe. Land, Development and the Future of Democratic Planning n, in European Stability Initiative, Berlin-Sarajevo, 12 décembre 2002.

${ }^{12}$ Stabilisation Force, force de stabilisation militaire internationale sous mandat de l'O.N.U. 
matériel de construction ont été distribués essentiellement entre 1998 et $1999^{13}$, ce qui correspond au début du retour des Bochniaques et des Serbes en Herzégovine, en particulier autour de Mostar et au sud de la ville. Cette période a été marquée par des tensions exacerbées entre les différentes communautés : 92 maisons de familles de retour ont été détruites au courant du premier semestre 1998 dans le canton Herzégovine-Neretva ${ }^{14}$. D'après l'OHR, le taux de vétérans croates de Bosnie-Herzégovine parmi ces nouveaux habitants serait élevé. Les villas de ces hameaux sont imposantes : deux ou trois étages, un appartement entier à chaque étage, et les fondations, impressionnantes par leur profondeur, ressemblent à des bunkers, comme si les villas avaient été construites pour pouvoir résister à une prochaine guerre.

Tous les déplacés ne sont pas si bien lotis et certains vivent encore dans des camps de fortune. Ainsi, 80 déplacés bochniaques installés à Blagaj (10 kilomètres au sud-est de Mostar) vivent dans des conditions sanitaires épouvantables : entassés depuis plusieurs années dans cinq containers, sans eau courante ni électricité, sur le bord d'une route de terre battue sillonnée par les blindés de la SFOR. Plus au sud, 15 o déplacés croates vivent dans 75 wagons immobilisés à Čapljina, fortement touchés par le chômage et sans espoir de pouvoir retourner chez eux.

L'exemple concret de deux familles déplacées à Mostar, l'une bochniaque et l'autre croate, montre les difficultés d'intégration auxquelles elles sont confrontées, malgré l'homogénéité ethno-nationale de l'environnement dans lequel elles évoluent. Les Dizdarević sont bochniaques, ils habitaient à Čapljina, une petite ville située à une trentaine de kilomètres au sud de Mostar, déjà à majorité croate avant la guerre. L'époux a passé de longs mois dans un camp de prisonniers croate, puis il a été libéré et envoyé en Norvège grâce à la Croix Rouge Internationale. Sans nouvelles pendant des mois de sa femme et de ses deux jeunes enfants, entre-temps expulsés et réfugiés à Mostar, il a décidé de rentrer au pays avant la fin du conflit pour retrouver les siens et s'est engagé dans l'armée de Bosnie-Herzégovine un an avant la fin de la guerre. Ils ont changé de domicile à de nombreuses reprises à Mostar, chaque fois obligés de restituer leur logement au propriétaire initial et de changer les enfants d'établissement scolaire, leur laissant chaque fois à peine le temps de s'insérer dans le réseau social du voisinage. Ingénieur en agronomie, le mari supervise un projet de développement agricole pour une ONG alors que la femme qui était fonctionnaire dans l'administration est sans emploi. Ils sont combattus

\footnotetext{
${ }^{13}$ Selon les estimations des autorités du canton 7 (Herzégovine-Neretva) de mai 1999, 2843 parcelles individuelles avaient été distribuées, réunies en 19 nouveaux hameaux, 1500 des parcelles étaient en phase de construction.
}

${ }^{14}$ Canton 7 dont Mostar est le chef-lieu; en 2002, le nombre de parcelles allouées est de 4200 et le taux de construction de $90 \%$ (OHR, art.cit.). 
entre l'impatience de la restitution de leur appartement à Čapljina où ils ne sont encore jamais retournés, et la crainte de s'y réinstaller tant que la ville est aux mains des nationalistes croates. L'époux ne veut plus entendre parler d'amitié avec les Croates : " Nous avons l'impression que toutes les bonnes relations que nous avons eu dans le passé étaient fausses et qu'ils n'attendaient que le moment opportun pour montrer ce qu'ils sont réellement "15. Leurs relations sociales sont assez limitées d'après le mari, elles se sont essentiellement confinées au noyau familial.

Les Sivić sont des déplacés croates, originaires de Konjic. La famille est composée d'un couple d'une quarantaine d'années, d'une fille de 20 ans et d'un fils adolescent scolarisé. Le mari était soldat dans l'armée croate HVO (Hrvatsko Vijeće Obrane) et s'est retrouvé sans emploi à la fin de la guerre. Il travaille irrégulièrement, sa femme est à la maison, de même que la fille aînée qui ne travaille pas non plus. Ils ne rencontrent jamais personne, si ce n'est la voisine immédiate, propriétaire de la maisonnette qu'ils louent à quelques kilomètres de Mostar, avec laquelle l'épouse prend quotidiennement le café. Comme ils ont rarement assez d'argent pour payer le loyer, chaque fois qu'il est nécessaire (fauche des foins, vendanges, etc.), ils aident les propriétaires qui vivent grâce à leur terre, à la vigne, et à quelques bêtes. En réalité, les propriétaires n'ont pas vraiment choisi de louer leur maisonnette :

Un Croate qui s'occupait des réfugiés est venu ici et a fait la liste de toutes les maisons inoccupées et nous a dit qu'il fallait aider les réfugiés; en fait ils nous ont demandé d'héberger cette famille... oui, c'est normal car les pauvres, ils ont tout perdu, ils étaient sans logement, mais je préfèrerais n'avoir personne ici car ils n'entretiennent pas du tout la maison et en plus, je sais que la situation est difficile car c'est pour tout le monde la même chose, mais ils n'arrivent presque jamais à payer le loyer, pourtant il n'est pas élevé, c'est juste pour dire qu'il y a un loyer, et en plus ils ne sont pas très propres ${ }^{16}$.

L'épouse assiste régulièrement à la messe quotidienne de l'église du village avec la propriétaire, mais sa participation à la vie religieuse n'a pas pour autant facilité son intégration dans le réseau de relations sociales du village. Ils n'ont pas eu la chance d'obtenir un terrain pour construire une maison et ne souhaitent pas non plus retourner à Konjic, même si les parents de l'épouse y résident encore.

Parmi les nombreux problèmes auxquels les déplacés se trouvent confrontés figurent le chômage et le risque d'expulsion des logements qu'ils

\footnotetext{
15 Omer, 45 ans.

${ }^{16}$ Vera, croate, 50 ans.
} 
occupent illégalement depuis la guerre. La situation économique de la Bosnie-Herzégovine a encore empiré depuis le départ de la plupart des ONG vers le Kosovo et l'arrêt du programme mondial contre la faim des Nations Unis en juin 1999. Seuls ceux qui ont des membres de la famille réfugiés à l'étranger vivent un peu mieux que les autres. À Mostar, presque la totalité de l'importante implantation industrielle a été détruite pendant la guerre et la ségrégation ethno-nationale fait loi sur le marché de l'emploi. Ce sont surtout les projets dans le domaine agricole qui ont bénéficié du financement des ONG alors que les usines et les industries peinent à être reconstruites. Le plus important complexe industriel qui fonctionne encore, Aluminij ${ }^{17}$, a été réparé grâce à des investissements étrangers (croate, allemand, suisse, américain) ; il est contrôlé par les Croates et n'emploient plus qu'un cinquième du personnel d'avant guerre, raison supplémentaire pour les dirigeants croates d'entraver les manœuvres de la communauté internationale pour le retour à l'emploi des anciens ouvriers bochniaques et serbes. La situation est identique avec l'industrie aéronautique et automobile Soko dirigée par les Croates, hormis qu'elle fonctionne à très bas régime depuis la guerre. Côté bochniaque, seule une petite usine d'habillement et l'usine de tabac partiellement détruite sont encore en activité, mais cette dernière ne fait plus travailler qu'une centaine de personnes. Le taux de chômage atteint les 20 \% à l'ouest et les $60 \%$ à l'est.

Les déplacés bénéficiant d'une formation professionnelle se sont insérés plus facilement dans le monde du travail, remplaçant les postes vacants des nombreux Mostariens réfugiés à l'étranger. En revanche ceux qui étaient sans qualification sont fortement touchés par le chômage et survivent tant bien que mal grâce à de petits travaux ponctuels. Du côté croate, les Mostariens déplorent souvent que des déplacés aient un emploi alors qu'eux-mêmes ont perdu le poste qu'ils occupaient avant la guerre et que les jeunes trouvent difficilement du travail. Pour les nationalistes croates, offrir un emploi à un déplacé plutôt qu'à un Mostarien participe de la politique de non retour des déplacés, afin de maintenir des zones à majorité, voire exclusivement croates. Côté bochniaque, les anciens habitants constatent avec amertume la même situation : " Ce sont des réfugiés qui ont eu les emplois à la poste, dans l'administration, alors que beaucoup de Mostariens sont sans emploi (...). C'est comme à Sarajevo, là ce sont les gens du Sandjak qui ont pris le pouvoir et les Sarajéviens tombent toujours plus bas (...). La plupart des gens qui dirigent ici ne sont pas de Mostar, beaucoup de policiers aussi ne sont pas d'ici "18.

Ce discours des habitants de Mostar révèle plus leur animosité envers les

${ }^{17}$ La compagnie réalise $25 \%$ du total des exportations de la Bosnie-Herzégovine (Banque Mondiale 2002).

${ }^{18}$ Lejla, bosniaque, 30 ans. 
déplacés qu'il ne reflète la réalité, car une enquête réalisée par la Banque Mondiale en février 2002 montre que le taux de chômage est supérieur chez les déplacés que chez les résidents d'origine (réfugiés de retour inclus) ${ }^{19}$. Ils font partie des personnes les plus vulnérables, $45 \%$ des déplacés en Fédération bosno-croate appartient à la catégorie des " très pauvres ".

Le logement est un autre problème majeur qui ralentit le processus de retour des déplacés. Lorsque après dix ans, les gens se sont habitués à un certain confort urbain, même s'ils vivent encore souvent dans des conditions précaires, ils n'ont guère envie de retourner vivre à la campagne. Les enfants aussi refusent souvent de quitter leurs amis et la ville dans laquelle ils ont grandi. Et, quand bien même leur habitation a été reconstruite grâce à l'aide humanitaire internationale, selon un modèle de confort urbain, les déplacés sont encore réticents à rentrer chez eux. Les cas de double occupation ne sont pas exceptionnels, c'est-à-dire que des personnes occupent un logement qui n'est pas le leur, refusant de retourner dans leur habitation reconstruite ou restituée qu'ils louent parfois à d'autres déplacés.

Mes anciens voisins bosniaques habitent maintenant dans ma maison qui était plus belle que la leur et ils louent leur maison à des déplacés bochniaques. ${ }^{20}$

C'est un criminel croate qui occupe mon appartement, il a un appartement à Konjic, une garçonnière à Mostar et je n'arrive pas à obtenir qu'il soit délogé de mon appartement... heureusement que nous avons cette maison de famille, mais autrement, comment aurais-je fait ? ${ }^{21}$

Le petit village bosniaque de Podvelež (situé à une demi-heure de route, dans la montagne à l'est de Mostar) est significatif de cette situation : il a été entièrement reconstruit en 1998, avec un confort sanitaire qui n'existait pas auparavant, mais ses habitants réfugiés à Mostar préfèrent rester à la ville. Les jeunes couples qui ont des enfants ne peuvent pas retourner au village, faute d'école et de ligne de transport régulière pour Mostar, d'autant que la seule perspective de travail reste l'agriculture ; par conséquent seules quelques personnes âgées sont rentrées et de nombreuses maisons toutes neuves restent inhabitées. Les jeunes refusent catégoriquement de suivre leurs parents pour labourer une terre caillouteuse, d'autant que la ville offre beaucoup plus de divertissement et un réseau de relations sociales plus étendu. Ce sont les déplacés âgés qui désirent le plus rentrer chez eux.

${ }^{19}$ Le taux de chômage atteint les $37,6 \%$ auprès des déplacés contre $24,5 \%$ chez les résidents d'origine. Une enquête antérieure de la banque Mondiale a comptabilisé $66 \%$ de chômeurs parmi les déplacés et les réfugiés de retour en 1999.

${ }^{20} \mathrm{Nada}$, croate, 45 ans.

${ }^{21}$ Emina, bochniaque, 65 ans. 
Deux raisons principales expliquent la réticence des déplacés devant la perspective du réintégrer leur habitation d'avant guerre. La première résulte de l'expérience traumatisante de la fuite comme seul moyen de survie : le déplacement a eu lieu pendant le conflit, dans un contexte de conquête du territoire par un autre groupe ethno-national. La seconde tient à ce que les conditions de sécurité ne sont pas garanties pour les déplacés de retour qui se retrouvent en position de minorité aux côtés d'une population potentiellement hostile. Le retour est donc synonyme de pauvreté et de racisme, de grandes difficultés à trouver du travail, de limitation à une agriculture de survie, de scolarisation des enfants dans une école où l'enseignement est dans la langue de l'" Autre " et où les manuels scolaires racontent l'histoire selon le point de vue adverse.

La pauvreté grandissante ne permet plus aux individus d'offrir l'hospitalité qui était de règle, ce qui a contribué à affaiblir les liens sociaux ${ }^{22}$. Les déplacés vivent repliés sur eux-mêmes et leurs rapports sociaux se limitent à la fréquentation d'autres déplacés du même village, voire strictement aux relations intra-familiales. Les relations de voisinage, komšiluk, autrefois à la base de la société se sont rarement reconstituées, si ce n'est parfois en milieu rural, en partie à cause de l'incompréhension culturelle et d'un certain mépris réciproque entre urbains et ruraux.

$80 \%$ des gens ont changé à l'est, on ne reconnaît plus personne. Les gens de Mostar me manquent, ils me manquent beaucoup. Ils sont complètement différents des gens de Mostar, tu le vois, ils crachent dans la rue, ils jettent les saletés par terre, c'est normal qu'ils ne respectent pas le lieu car il n'est pas à eux; en plus, ils laissent les chaussures sur le pas de la porte dans la cage d'escalier, comme à la campagne $!^{23}$

Ici, ils se croient encore à la campagne, parfois ils jettent les ordures par la fenêtre du haut des immeubles. Ils ne tiennent pas les appartements en bon état, de toutes façons, ils ne sont pas à eux, et ils refusent de participer pour payer quelqu'un qui vienne nettoyer les parties communes, maintenant tout est sale. ${ }^{24}$

\footnotetext{
${ }^{22}$ En 2003, la Banque Mondiale estimait à $20 \%$ le taux de personnes vivant en-dessous du seuil de pauvreté ( $25 \%$ en République serbe et $16 \%$ en Fédération bosno-croate) et $30 \%$ le taux de personnes vulnérables susceptibles de glisser en dessous du seuil de pauvreté. Tous les habitants s'accordent à dire que les conditions de vie étaient meilleures à la fin de la guerre grâce à l'aide humanitaire alimentaire ; aujourd'hui le taux de chômage est encore très élevé et l'économie est toujours en grande partie dévastée (l'implantation industrielle a été presque totalement détruite pendant la guerre et les entreprises qui ont repris leur activité ne fonctionnent plus qu'avec en moyenne 10 à $20 \%$ du personnel d'avant guerre) .
}

${ }^{23}$ Amna, bochniaque, 30 ans.

${ }^{24}$ Maja, croate, 35 ans. 
Non seulement les déplacés vivent dans des conditions matérielles difficiles, mais en plus ils sont nombreux à souffrir de troubles psychosomatiques engendrés par la guerre et aggravés par le statut même de déplacé : le fort taux de chômage et l'origine rurale ne leur permet pas de s'insérer dans un réseau de relations sociales extra-familiales, ils risquent à tout moment d'être expulsés de leur logement, la plupart d'entre eux sont coupés de leur milieu social, les liens de parenté sont dissolus voire rompus par l'éparpillement des familles ou la perte des proches pendant le conflit, leur dignité est entachée par la précarité matérielle (par exemple, ils ne sont plus à même d'offrir l'hospitalité traditionnelle exacerbée dans cette région). Cette situation a entraîné l'augmentation des violences conjugales dans les familles de déplacés. La guerre a enfermé chacun dans sa souffrance et dressé des barrières là où régnait autrefois le komšiluk, les relations entre voisins des différentes communautés sous forme de dons et contre-dons de services et de nourriture à l'occasion des fêtes religieuses, des naissances et des enterrements.

La communication avec des déplacés est entravée par la douleur que réveille la narration des conditions de l'exode et par la suspicion à l'égard de l'étranger. Ils sont peu loquaces lorsqu'ils sont originaires de petits villages, les plus bavards sont les individus d'un niveau social plus élevé qui occupaient des emplois administratifs dans de petites villes. Les représentants des communautés de déplacés usent habilement de la langue de bois et leur discours est conditionné par le contexte d'un pays sous tutelle de la communauté internationale, où tout Européen est un collaborateur potentiel des "occupants ". La communauté internationale est relativement tolérée par les Bochniaques puisqu'elle a imposé la paix et leur a attribué des territoires conquis par les Croates et les Serbes, alors que les Croates lui sont ouvertement hostiles, car l'arrêt de la guerre a mis un terme à leur expansion territoriale et entravé leurs manœuvres séparatistes. Les conflits d'intérêts économiques et territoriaux qui se déroulent désormais sur le plan politique conditionnent les discours des uns et des autres selon les directives des partis nationalistes au pouvoir. Le contraste entre les propos tenus par les différents acteurs et la réalité observable sur le terrain met en évidence l'instrumentalisation politique de la question des déplacés. Avec les entretiens et l'observation des pratiques, les cartes mentales réalisées par des jeunes, consistant à dessiner Mostar, révèlent à quel point les déplacés sont confinés dans la partie de la ville où ils résident, l'autre partie représentant en quelque sorte un monde étranger, territoire de l'" Autre ", lieu de danger potentiel jamais exploré25.

Trahis par leur accent, ils ne passent pas inaperçus auprès des anciens habitants de Mostar. Ils se distinguent aussi par la tenue vestimentaire, hormis

25 Chacun ne représente que la partie de la ville dans laquelle il réside. 
les enfants et les adolescents qui ont grandi à la ville au contact des petits urbains. Ce sont surtout les femmes bochniaques d'origine rurale qui se reconnaissent au premier coup d'œil, car un bon nombre de femmes d'âge mûr portent les dimje (pantalons bouffants d'origine turque) et couvrent leurs cheveux d'un foulard bariolé, comme il est d'usage à la campagne. C'est aussi leur visage qui trahit les déplacés, car la ville fonctionne comme un gros bourg où les résidents des différents quartiers se connaissent tous de vue.

La différence entre les mondes rural et urbain était nettement marquée en ex-Yougoslavie. Alors que la majorité des réfugiés est d'origine urbaine, cosmopolite et plus éduquée (degré d'instruction), leurs concitoyens déplacés sont principalement d'origine rurale, moins éduqués et habitués à un environnement plus homogène, ce qui provoque des tensions entre urbains et ruraux déplacés, accentuées par la relative étanchéité entre ces deux mondes avant la guerre. Le terme seljak / seljaci qui signifie " paysan " a une forte connotation péjorative et sous-entend " inculte / arriéré "; les ruraux sont aussi affublés du qualificatif péjoratif de primitivni, " primitifs ". La population rurale et provinciale est plus traditionnelle et fortement endogame. En revanche, la ville est symbole de mixité et d'échange intercommunautaire, l'espace de la modernité et le lieu d'exercice du pouvoir ${ }^{26}$. L'écart culturel entre urbains et ruraux crée un fossé peut-être plus important que celui qui pouvait exister entre Bochniaques, Croates et Serbes de la même ville, qui partageaient un style de vie commun, avaient grandi dans les mêmes écoles, travaillaient ensemble, voire s'épousaient. Déracinés brutalement de leur espace rural au cours de la guerre, les déplacés se sont retrouvées en décalage culturel et matériel avec les habitants de la ville.

Le discours des Mostariens, aussi bien croates que bochniaques, sur les déplacés est récurrent : " trop contents d'avoir quitté la vie dure de la campagne, ils préfèrent se contenter de survivre à la ville ». La secrétaire d'un des départements de l'université de Mostar ouest, croate, a d'ailleurs remarqué que les étudiants déplacés d'origine rurale obtiennent de meilleurs résultats à l'université que les étudiants de Mostar, car " ils savent bien que s'ils ne réussissent pas, ce qui les attend de retour chez eux, c'est la bêche ".

Les Mostariens, qu'ils soient ou non de la même communauté ethno-nationale, ont le sentiment d'avoir été envahis et dépossédés de leur propre ville par les déplacés ruraux, oubliant que la ville s'est agrandie avec l'exode rural et les besoins en main-d'œuvre de l'industrie en plein essor, au lendemain de la Seconde Guerre mondiale. Même si cela participe du mythe nationaliste de

${ }^{26} \mathrm{Cf}$. Chaslin (François), Une Haine monumentale. Essai sur la destruction des villes en ex-Yougoslavie, Paris : Descartes et Cie, 1997. 
" revanche des campagnes "27 sur les villes, c'est néanmoins à travers ce prisme que les habitants de Mostar interprètent aujourd'hui la réalité qui les entoure. Ils déplorent qu'au lieu de s'adapter au mode de vie et à " la mentalité plus ouverte du monde urbain ", les déplacés aient apporté leur " mentalité arriérée " qui entache leur Mostar et continuent de se comporter comme chez eux, malgré les années passées dans la ville. Certains Mostariens rejettent la responsabilité de la division de leur ville sur les déplacés ruraux, moins habitués à vivre dans un environnement mixte, sans lesquels, pensent certains, le fossé entre Croates et Bochniaques de Mostar serait de moindre importance.

\section{LE RETOUR : L'ÊTRE MINORITAIRE ET SES DIFFICULTÉS}

L'annexe 7 des accords de Dayton relative aux réfugiés et aux déplacés stipule le droit au libre retour dans le lieu de résidence d'origine et à la restitution des biens, le droit à la protection contre toute tentative d'intimidation, de persécution ou de discrimination relative à l'appartenance ethnique, à la croyance religieuse ou à l'opinion politique. Malgré cela le processus de retour est demeuré lent et difficile jusqu'en 2001, en particulier dans les villes, autrefois mixtes : d'une part beaucoup de logements sont occupés par d'autres déplacés, et d'autre part, déplacés et réfugiés voient leurs tentatives de retour très souvent enrayées par les dirigeants politiques locaux nationalistes, issus de la majorité ethno-nationale. Les politiciens nationalistes serbes et croates opèrent dans le sens du maintien de territoires le plus homogènes possible. La politique séparatiste leur garantit de rester au pouvoir et de contrôler les ressources économiques locales en maintenant les privilèges d'une entité politique autonome pour les Serbes (la République serbe) et en revendiquant la création d'une troisième entité politique autonome ou la partition du pays en cantons à majorité ethnique pour les Croates. Par conséquent, les retours en République serbe ainsi que dans les villes à majorité croate du territoire autoproclamé République croate d'Herzeg-Bosnie pendant la guerre sont jusqu'à ce jour moins nombreux et plus sensibles que dans les zones à majorité bochniaque. Les dirigeants bochniaques se montrent en effet plus enclin à suivre la politique de retour des déplacés vivement encouragée et récompensée par la communauté internationale qui favorise le financement des projets de reconstruction et de développement multiethniques. Au contraire des Croates et des Serbes de Bosnie-Herzégovine qui bénéficient du soutien d'un État voisin auquel les nationalistes croates et serbes souhaitaient annexer les territoires de la Bosnie-

27 Cf. Bougarel (Xavier), "La "revanche des campagnes" : entre réalité sociologique et mythe nationaliste n, Balkanologie, 2 (1), juillet 1998. 
Herzégovine qu'ils se seraient partagés pendant la guerre, les Bochniaques n'ont pas d'autre mère patrie que la Bosnie-Herzégovine vers laquelle se tourner. C'est pourquoi, depuis la déclaration d'indépendance en mars 1992, ils ne cessent de lutter pour le maintien de l'intégrité territoriale du pays et pour la défense de sa multiethnicité par l'intermédiaire du SDA (Stranka Demokratske Akcije - Parti de l'Action Démocratique) fondé par Alija Izetbegović. Les nationalistes bochniaques qui souhaitent éviter un second découpage ethnique du territoire de la Fédération entre Bochniaques et Croates et réviser les accords de Dayton pour une réunification de la Bosnie-Herzégovine (en supprimant l'entité serbe autonome) favorisent le retour de tous les déplacés bochniaques. Dans ce contexte, le vote des déplacés est un enjeu politique important au point que les déplacés serbes et croates votent massivement dans leur commune d'adoption, selon les directives des partis nationalistes, tandis que les Bochniaques votent majoritairement dans leur commune d'origine pour compenser le pouvoir des nationalistes de l'opposition dans les régions ethniquement plus homogènes. Les premiers Serbes ont commencé à rentrer chez eux dès juin 1998, mais les retours de Serbes sont encore exceptionnels. Les Croates sont également très peu nombreux à rentrer, influencés par les exhortations de leurs représentants politiques à rester dans leur nouveau lieu de résidence.

Pour accélérer le retour des déplacés et des réfugiés, la communauté internationale a adopté des mesures drastiques par l'intermédiaire d'une nouvelle loi sur le retour, promulguée en 1999. L'objectif est d'obliger les dirigeants locaux à établir des services efficaces chargés des logements, de permettre aux tribunaux d'être indépendants, de construire des logements supplémentaires là où il est nécessaire, d'organiser les retours sous protection des forces internationales de maintien de la paix. Cette loi prévoit une amende pour refus de coopération de la part des autorités locales, voire le licenciement des fonctionnaires qui entravent le processus de retour, un délai maximum de 90 jours pour traiter les réclamations et les requêtes soumises aux services chargés des logements, un délai de 15 à 90 jours à la police pour expulser les occupants illégaux (y compris manu militari) et un délai maximum de go jours pour retourner dans les logements à la date d'obtention des permis, sous peine de perte définitive des droits relatifs à la restitution du logements. Les résultats se sont montrés probants puisque le processus de retour des déplacés et des réfugiés s'est nettement accéléré depuis la fin de l'année $2000^{28}$.

${ }^{28}$ Les estimations de l'UNHCR sont les suivantes pour l'ensemble du territoire de la Bosnie-Herzégovine : 01/03/1999 : 836500 déplacés, 31/08/1999 : 838 000 déplacés, 31/08/2000: 793500 déplacés, 10/2001 : 470500 déplacés, 04/2002: 414500 déplacés, 12/2002 : 375000 déplacés. 
En réponse à la pression accrue de la communauté internationale, les incidents interethniques se sont multipliés en République serbe, dans le canton Herzégovine-Neretva de Mostar et à Sarajevo, ainsi que les tentatives d'intimidation des déplacés de retour et les actes de violence ponctuels contre les édifices religieux. Ces tentatives d'intimidation ont pour but de dissuader les candidats au retour, déjà effrayés à l'idée que de nombreux criminels de guerre sont toujours en liberté et que la police est mono-ethnique. Des émeutes ont eu lieu lors de l'inauguration de deux mosquées à Trebinje et à Banja Luka en République serbe en mai 2001, un incendie criminel a détruit la palissade délimitant l'espace de reconstruction prochaine d'une des mosquées de Stolac fin 2001, sans compter les atteintes répétées à l'intégrité physique des personnes de retour allant parfois jusqu'au meurtre ${ }^{29}$. À cela s'ajoute le problème des nombreuses mines et explosifs dispersés sur tout le territoire ${ }^{30}$, spécialement autour des anciennes lignes de front, qui menacent les déplacés de retour dans les zones rurales et freinent la reprise de l'activité agricole.

La situation s'est quelque peu améliorée à Mostar, en particulier depuis l'année 2000, avec la restitution progressive des habitations à leurs propriétaires d'avant-guerre, bien que ce soient surtout les Bochniaques qui retournent habiter à l'ouest alors que très peu de Croates retournent à l'est. Les petites annonces offrent beaucoup plus de propositions d'échanges de maisons et d'appartements que de propositions de vente, ce qui montre combien Bochniaques et Croates sont méfiants quant à la perspective de retourner dans leur logement d'avant-guerre et préfèrent conserver le plus possible l'homogénéité ethno-nationale de leur environnement quotidien.

Avant, j'habitais dans un appartement à Zalik, derrière la gare ; le propriétaire de cet appartement est bochniaque, il habite à l'étranger, en Suède, et il n'a pas l'intention de revenir ici, mais avant qu'il obtienne la restitution de son appartement pour le vendre et que nous soyons obligés de le quitter, nous allons essayer de nous mettre d'accord avec lui pour échanger notre ancien appartement avec celuici (...) je n'ai pas envie de retourner vivre là-bas, avant c'était autre chose, mais maintenant il vaut mieux que ce soit chacun chez soi. ${ }^{31}$

\footnotetext{
29 Une jeune fille bochniaque de retour avec sa famille a été tuée par balles dans leur maison reconstruite en République serbe en juillet 2001. Les grenades lancées contre des déplacés de retour ou les tirs de balles font partie des actes de violence sporadique.

${ }^{30} \mathrm{~A}$ la fin de la guerre, le territoire de la Bosnie-Herzégovine est parsemé d'environ 670 ooo mines et 650000 explosifs non explosés ( $12 \%$ des zones d'usage quotidien, de reconstruction et d'activités économiques sont minées). Entre 1996 et 2002 , les mines ont fait 1423 victimes dont 480 sont décédées. $35 \%$ des victimes sont des personnes de retour. Grâce au travail des organisations chargées du déminage, le taux d'accidents a baissé de 52 à 6 par mois de 1996 à 2002.
}

${ }^{31}$ Zdenka, croate, 40 ans. 
Le repli communautaire renforce le sentiment de sécurité et maintient les barrières élevées entre les communautés durant le conflit, fortifiées par les politiciens nationalistes au pouvoir.

De nombreuses organisations non gouvernementales qui étaient chargées de la distribution de l'aide alimentaire et matérielle n'assurent plus qu'une présence administrative à Mostar depuis 1999, suite à la fin du projet mondial contre la faim et au conflit serbo-kosovar qui offrait de nouvelles perspectives de financements. La majeure partie d'entre elles s'occupe de la reconstruction des habitations et de la réalisation de projets agricoles. Seule une ONG suisse se charge de l'assistance aux personnes déplacées de retour; entièrement gérée par du personnel local, elle travaille en collaboration avec l'UNHCR par l'intermédiaire d'une équipe de terrain mixte, bochniaquecroate. Son rayon d'action s'étend sur environ 30 kilomètres autour de Mostar. L'équipe est composée d'un psychologue et d'une assistante sociale chargés de se rendre auprès des déplacés de retours pour les informer de leurs droits et pour leur expliquer les démarches à suivre afin d'obtenir l'eau, l'électricité, les titres de transport gratuit, etc. Ils s'assurent aussi de leurs conditions de vie sociales, sanitaires, psychologiques et matérielles grâce à un long questionnaire. À la demande concernant la sécurité et d'éventuelles intimidations ou menaces, les gens répondent du bout des lèvres que "ça va ", mais qu'ils se sentaient plus tranquilles au début lorsque les militaires de la SFOR venaient chaque jour contrôler la situation. À la question portant sur les troubles du sommeil ou les divers symptômes, beaucoup souffrent en effet d'insomnies et de douleurs abdominales, de migraines, etc. Chaque fois, les personnes rencontrées se plaignent de la rareté des visites de l'équipe de terrain, seuls moments de communication, et s'excusent toujours platement de n'avoir rien à offrir à manger, tout juste un verre de sirop ou un café. Les déplacés de retour sont en proie à une extrême détresse morale et matérielle. Ce sont la plupart du temps des retraités, rarement des couples avec des enfants en âge de scolarisation ; leur lieu d'habitation se situe souvent à l'extérieur de la ville et inclut un peu de terrain cultivable. Deux principales raisons motivent en général leur retour : la nostalgie pour la terre natale pour les anciens et le chômage dans la ville où ils étaient réfugiés pour les jeunes : " Là où nous étions [dans la zone orientale de la République serbe], je ne trouvais pas de travail, je me sentais comme un étranger et les gens ne nous voyaient pas d'un bon œil, alors tant qu'à mourir de faim, je préfère mourir chez moi " $^{32}$.

Ils ont peu de ressources sur lesquelles compter, hormis un lopin de terre et parfois l'aide des militaires de la SFOR qui les avaient escortés au moment de la réintégration de leur ancienne habitation, c'est-à-dire quelques conserves 
et aliments secs de temps en temps. Ils ont tout perdu durant la guerre et reviennent comme ils étaient partis, avec une maigre valise contenant quelques vêtements. Ils sont d'autant plus coupés du monde qu'ils ne possèdent aucun moyen de locomotion et que l'obtention de titres de transport gratuit nécessite des démarches administratives auprès de leur municipalité de résidence, à majorité de l'autre groupe ethno-national, qu'ils ont souvent peur d'affronter. Le mobilier et le matériel de base est fourni par l'UNHCR : lits en bois grossièrement finis, quelques matelas et couvertures, réchaud à bois pour se chauffer et cuisiner, plastique pour calfeutrer les fenêtres. Certains ne sont pas encore retournés définitivement dans leur habitation, soit parce qu'elle n'est pas entièrement reconstruite, soit parce qu'ils n'ont ni les outils ni les semences nécessaires pour travailler la terre, soit parce que les champs n'ont pas encore été déminés. Les personnes âgées se retrouvent isolées du cercle familial car leurs enfants refusent de rentrer, parce qu'ils ont trouvé du travail ou parce qu'ils ne veulent pas scolariser leurs enfants dans des écoles de l'autre communauté ethno-nationale, par crainte de la discrimination.

On constate également que les maisons dans lesquelles les Serbes retournent sont rarement reconstruites au moment du retour, alors que celles des Bochniaques sont toujours presque entièrement refaites. En effet, le retour des Serbes n'est pas très bien accepté, ni par les Bochniaques ni par les Croates, ni par les autorités locales ni par les anciens voisins et amis qui leur attribuent la responsabilité de la guerre et les accuse d'avoir fui, parfois avant le déclenchement du conflit :

Comme s'ils avaient été avertis, car les Serbes pensaient qu'ils prendraient Mostar en quelques jours ; nos voisins serbes ne nous ont pas prévenus, mais ils sont partis en nous laissant les clés pour arroser les plantes, disant qu'ils partaient quelques jours chez des parents en Herzégovine orientale pour des vacances, et puis la guerre a commencé et ils ne sont pas revenus. Quand ma voisine serbe est rentrée et qu'elle a frappé à ma porte, je lui ai dit que je ne voulais pas la voir, elle s'est mise à pleurer, mais je lui ai dit " où étiez-vous pendant que nous étions sous les bombes ? Si vous étiez restés avec vos enfants, ils ne nous auraient pas bombardés comme ça pour ne pas tuer les Serbes $n .33$

Notre situation est très difficile, on n'a pas eu d'aide de la République serbe et très peu de la Fédération, tout va aux Bochniaques alors qu'eux reconstruisent déjà depuis 1994 et nous seulement depuis 1998. Notre retour a été instrumentalisé par les Bochniaques, moi j'étais côté croate, ils m'ont clairement dit qu'ils n'étaient pas pour le retour des Serbes et en général ils font obstruction; côté bochniaque, quand vous écoutez les médias, ils sont pour le retour, mais en réalité ça se passe 
tout autrement. La plupart des Serbes d'ailleurs sont retournés côté croate, les gens préfèrent peut-être retourner côté croate. Au début seuls les vieux retournaient chez eux, mais maintenant il y a de plus en plus de couples avec des enfants mais ça pose de nouveaux problèmes, sans parler du travail car il y a déjà beaucoup de chômeurs et en plus s'ajoute le problème de la nationalité pour être embauché. Nous, nous avons plus de chance car ma femme est croate et elle enseigne dans une école côté croate. 34

Dans le quartier de Raštani, rattaché à la municipalité ouest à majorité croate de Mostar, deux familles bochniaques ont réintégré leur maison à côté d'une famille serbe arrivée un mois plus tard. Les deux maisons bochniaques avaient été entièrement reconstruites et reliées au service des eaux de la municipalité, tandis que la famille serbe attendait toujours d'avoir l'eau courante dans une maison dévastée, dont seuls les murs et le toit tenaient encore, les fenêtres étaient calfeutrées par des plastiques de l'UNHCR, toute l'installation électrique avait été arrachée et les murs étaient encore noirs de l'incendie. Là vivaient depuis trois mois un homme, sa femme, leurs deux filles et la grandmère, dans un dénuement complet, totalement isolés et sans aucune ressource financière. Les Serbes rentrent peu à peu à Mostar, notamment dans la périphérie sud de la ville, à Ortiješ, Lakševina et Malo Polje, dans les hameaux où ils étaient autrefois majoritaires, dont les maisons systématiquement brûlées à leur départ n'avaient pas été réoccupées entre temps.

Il existe des retours spontanés, mais dans la plupart des cas ils sont organisés sous tutelle de l'UNHCR et sous escorte militaire de la SFOR pour plusieurs familles à la fois, de manière à assurer la protection d'une zone délimitée pendant les premiers temps. Les retours sont généralement plus nombreux en périphérie de Mostar qu'en ville, car les hameaux étaient avant la guerre déjà ethniquement homogènes, de même que les villages le plus souvent divisés en quartiers selon l'appartenance ethno-nationale ${ }^{35}$. Le retour organisé de plusieurs familles conjointement assure de vivre dans un environnement homogène et rassurant, au milieu de personnes connues, et appartenant à la même communauté ethno-nationale.

Une des caractéristiques principales de la situation des déplacés à Mostar, est l'opposition entre le poids de leur présence dans la ville et leur absence dans le tissu des relations sociales mostarien. Ils sont dans la ville, mais ils ne 
sont pas de la ville. L'appartenance ethno-nationale commune n'a visiblement pas permis de transcender la barrière de l'appartenance locale, accentuée par l'origine rurale. Ayant bien peu en commun, si ce n'est la tradition religieuse qu'ils vivent aussi différemment, les déplacés et les habitants d'origine n'entretiennent pas ou peu de relations sociales entre eux. Quand bien même ils vivent côte à côte, leurs relations de voisinage sont quasi inexistantes, malgré l'importance qu'elles revêtent dans cette société. Cela laisse-t-il entrevoir la possibilité de reconstruire la société civile au-delà des barrières érigées par la guerre entre les différentes communautés de la Bosnie-Herzégovine ? Bien sûr l'expérience extrême de la haine organisée, à travers l'emprisonnement dans les camps, les humiliations, la perte d'un parent, d'un époux ou d'un enfant, à travers la volonté de réduire l'Autre à des conditions de vie animales durant le conflit, à le déshumaniser, font partie des traumatismes profonds et des blessures longues à cicatriser, dont la mémoire se perpétue à travers les générations. La guerre a été certes traumatisante pour tous, mais plus encore pour ceux qui ont tout laissé derrière eux et dont la famille est désormais au mieux dispersée si ce n'est décimée.

Les nombreux problèmes sur le plan économique, politique, social, scolaire, etc., caractéristiques de la Bosnie-Herzégovine contemporaine ne favorisent pas le retour à Mostar et dans sa région des déplacés en âge de travailler, ni des familles dont les enfants doivent être scolarisés. C'est pourquoi ce sont encore principalement les personnes âgées qui réintègrent leur habitation d'avant-guerre, bénéficiant d'une maigre pension de retraite qui assure néanmoins une source de revenu minimum ${ }^{36}$.

La réunification de la ville sous tutelle de la communauté internationale n'a pas encore été réalisée car toutes les tentatives de restructuration dans ce sens sont systématiquement entravées par les leaders nationalistes, croates en particulier. Mostar symbolise désormais le pari de la communauté internationale qu'une Bosnie-Herzégovine intercommunautaire est encore possible. C'est pourquoi ses efforts pour réunifier la ville et reconstruire au plus vite le "vieux pont " sont considérables, comme si la restauration du patrimoine architecturale pouvait effacer des mémoires le souvenir de la guerre, en éliminer les conséquences au quotidien et Mostar retrouver sa tradition de tolérance légendaire en même temps que son nouveau " vieux pont ". La stabilisation à long terme de la situation du pays dépend en grande partie de l'orientation politico sociale de ville-clés comme Mostar. Mais, la crainte entretenue par le 
parti nationaliste croate $\mathrm{HDZ}-\mathrm{BiH}^{37}$ que les Croates soient un jour relégués au rang de minorité nationale explique le vote communautaire massif des Croates et la revendication incessante du découpage de la Bosnie-Herzégovine en cantons à majorité ethnique. Lors d'un entretien, un membre haut placé du HDZ-BiH a résumé en une phrase significative la " cause " des Croates de Bosnie-Herzégovine, défendue avec ferveur par leurs représentants politiques : " Nous Croates, l'histoire nous a appris que si nous voulions conserver notre culture et non nous voir imposer la culture d'un autre, comme ce fut le cas pendant 50 ans de communisme, nous devions nous imposer au niveau politique ".

Cependant cette explication culturaliste masque une partie de la réalité qui est celle de la défense des intérêts économiques des Croates en BosnieHerzégovine et la circulation des richesses au sein du canton ethnique où elles sont produites, plutôt que leur redistribution au niveau fédéral, comme à l'époque de la Yougoslavie communiste où le rendement des républiques les plus prospères permettaient d'aider les républiques les moins industrialisées et les plus pauvres.

Même s'il faudra encore du temps pour que tous les déplacés rentrent chez eux, le futur du pays est essentiellement tributaire de la reconstruction de l'économie et peut-être aussi d'un système scolaire commun, ce qui est en cours de réalisation ${ }^{38}$. La question des déplacés oppose les nationalistes serbes et croates à la communauté internationale. Celle-ci ne peut pas en effet accepter le découpage de la Bosnie-Herzégovine en cantons à majorité ethnique ${ }^{39}$ qui

37 Hrvatska Demokratska Zajednica-Bosna i Hercegovina, "Communauté Démocratique Croate de BosnieHerzégovine ", il est le prolongement de la HDZ de Croatie fondé par l'ancien président croate Franjo Tudjman, et a été longtemps présidé par Mate Boban. Le projet de la HDZ-BiH pendant la guerre était d'annexer à la mère patrie croate cette région peuplée majoritairement de Croates ; aujourd'hui le parti revendique la création d'une troisième entité politique croate en Bosnie-Herzégovine qui correspondrait au territoire d'Herzégovine occidentale (autoproclamée République d'Herzeg-Bosnie pendant la guerre en 1993).

$3^{8}$ Théoriquement, les èlèves peuvent s'inscrire dans toutes les écoles, indépendamment de leur origine ethno-nationale, mais en réalité les écoles de Bosnie-Herzégovine sont soumises à un régime d'apartheid. Les écoles situées dans les municipalités à majorité croate étudiaient à partir de manuels en langue croate et de programmes scolaires de Zagreb, ceux des municipalités à majorité bochniaque en langue bosniaque à partir des manuels et du programme de Sarajevo et celles des municipalités à majorité serbe à partir de manuels en langue serbe et du programme de Belgrade. Les manuels d'histoire traitaient des derniers évènements de la guerre selon trois points de vue différents et peu objectifs et ceux de littérature se contredisaient sur la nationalité de certains auteurs. Une première commission d'experts locaux et internationaux a retiré les manuels scolaires " dangereux " publiés par Zagreb et Belgrade, susceptibles de renforcer les clivages et d'inciter à la haine entre les jeunes générations. En 2003, une nouvelle commission d'experts mixte a été réunie pour éliminer les éléments qui pourraient offenser ou mettre dans l'embarras les personnes de l'autre groupe ethno-national, et s'accorder sur l'enseignement de la période ottomane et de la nationalité de certains auteurs.

39 Même si en réalité la division de la Bosnie-Herzégovine en cantons ethniques est une solution qu'avait envisagée la communauté internationale en proposant l'adoption par les parties en conflit d'abord du plan Vance-Owen en janvier 1993, puis du plan Owen-Stoltenberg en juillet 1993. 
légitimerait le principe du nettoyage ethnique. De leur côté, les dirigeants nationalistes serbes et croates poursuivent le but de la guerre par l'intermédiaire des déplacés, c'est-à-dire qu'ils tentent de conserver le contrôle de zones à majorité ethnique en entravant le retour des déplacés qui menacerait la pérennité de leur pouvoir. L'exemple de Mostar montre aussi que l'appartenance ethnonationale commune n'a pas permis de tisser une nouvelle trame sociale entre anciens et nouveaux Mostariens. La situation des déplacés illustre à la fois les difficultés à rétablir une paix véritable dans un pays sorti de la guerre depuis 8 ans bientôt et combien la signature de la paix n'éradique pas d'un jour à l'autre la violence imposée par la guerre : "On peut parler de paix mais il s'agit d'une paix imposée de l'extérieur. Moi je la définis une paix froide par analogie avec la guerre froide. En Bosnie aujourd'hui, c'est un concept négatif de la paix qui prévaut, que l'on pourrait définir de façon kantienne comme une situation d'absence de guerre ${ }^{\circ} 4^{\circ}$. 